\title{
Onset Detection in Surface Electromyographic Signals: A Systematic Comparison of Methods
}

\author{
Gerhard Staude \\ Institut für Mathematik und Datenverarbeitung, Universität der Bundeswehr München, Germany \\ Email: gerhard.staude@unibw-muenchen.de \\ Claus Flachenecker \\ Institut für Mathematik und Datenverarbeitung, Universität der Bundeswehr München, Germany \\ Email: claus.flachenecker@humphrey.com

\begin{abstract}
Martin Daumer
Institut für Medizinische Statistik und Epidemiologie, Technische Universität München, Germany Email:martin.daumer@imse.med.tu-muenchen.de

Institut für Mathematik und Datenverarbeitung, Universität der Bundeswehr München, Germany Email: Werner.Wolf@unibw-muenchen.de
\end{abstract} \\ Werner Wolf
}

Received 26 July 2000 and in revised form 13 May 2001

\begin{abstract}
Various methods to determine the onset of the electromyographic activity which occurs in response to a stimulus have been discussed in the literature over the last decade. Due to the stochastic characteristic of the surface electromyogram (SEMG), onset detection is a challenging task, especially in weak SEMG responses. The performance of the onset detection methods were tested, mostly by comparing their automated onset estimations to the manually determined onsets found by well-trained SEMG examiners. But a systematic comparison between methods, which reveals the benefits and the drawbacks of each method compared to the other ones and shows the specific dependence of the detection accuracy on signal parameters, is still lacking. In this paper, several classical threshold-based approaches as well as some statistically optimized algorithms were tested on large samples of simulated SEMG data with well-known signal parameters. Rating between methods is performed by comparing their performance to that of a statistically optimal maximum likelihood estimator which serves as reference method. In addition, performance was evaluated on real SEMG data obtained in a reaction time experiment. Results indicate that detection behavior strongly depends on SEMG parameters, such as onset rise time, signal-to-noise ratio or background activity level. It is shown that some of the threshold-based signal-powerestimation procedures are very sensitive to signal parameters, whereas statistically optimized algorithms are generally more robust.
\end{abstract}

Keywords and phrases: EMG, electromyography, onset detection method, performance, comparison.

\section{INTRODUCTION}

Analysis of electromyographic signals recorded from the skin over the muscles by surface electrodes (SEMG) represents an important tool in a variety of applications like neurological diagnosis, neuromuscular and psychomotor research, sports medicine, prosthetics, or rehabilitation. Processing of SEMG (and other biosignals like EEG, ENG, ECG, etc.) can be considered a special field of applied signal processing, with the main focus on an appropriate application of theoretical concepts to extract specific information from small and often noisy biosignals.
The instantaneous SEMG signal energy serves as a measure of the current level of muscle activation [1], whereas the determination of the exact onset and offset times of a muscle contraction is useful in studies of motor control and performance $[2,3,4,5,6,7,8,9,10]$. The latter is of particular importance in reaction time (RT) experiments, and numerous methods were developed for SEMG onset detection. Although a comparison of results obtained with various approaches was presented $[11,12,13]$, an objective and comprehensive assessment of their accuracy in SEMG onset detection is still lacking. Due to the fact that the underlying signal parameters including the "true" onset and offset 
times are unknown in real SEMG recordings, a rating by an objective error measure is not possible. Therefore, the automatically determined onsets are usually compared to results obtained by visual inspection of the data by some well-trained SEMG examiner. But a profound performance test should be based upon a relevant statistical analysis which requires a large amount of SEMG data with properly preset signal parameters. This analysis is difficult or even impossible with real data due to the poor parameter reproducibility of successive SEMG recordings, and due to inter- and intra-rater variability introduced by the manually determined reference onset estimates. To overcome this problem, simulated SEMG data were used in this study for assessing the sensitivity of various detection methods to changes in signal parameters. Also, the structure of the various existing SEMG onset detection methods was analyzed revealing several common components which are identical in a logical way, but are formulated individually by different authors. As basis for comparison, some fundamental algorithmic principles formulated in [12] were used to express each method by these terms.

Detection algorithms introduced earlier by Hodges and Bui [13], Bonato et al. [14], Lidierth [15], Abbink et al. [2], and two new implementations of the model-based detector proposed by Staude et al. [12] are included in this study. The algorithmic layout of the methods is compared, and the dependence of onset detection accuracy on signal parameters is demonstrated. The use of a statistically optimal estimator as a reference for the upper performance limit allows an absolute performance ranking of each method. In addition, the methods were tested on real SEMG data obtained in a reaction time situation.

\section{ONSET DETECTION ALGORITHMS}

\subsection{General scheme of onset detection}

As suggested in [12], the comparison of algorithmic structures is conducted by using the SEMG signal model and general scheme of event (onset) detection shown in Figure 1. The digitized SEMG signal may be represented by a real-valued sequence $\left(x_{k}\right)_{k \geq 1}$, where $x_{k}$ denotes the signal amplitude at a particular discrete time instant $k$; thus a single SEMG record $\left(x_{k}\right)_{k \geq 1}$ represents a sample observation of a discrete random process (a zero mean discrete white Gaussian noise process) $\left(W_{k}\right)_{k \geq 1}$ exciting a linear system with transfer function $H(z) .\left(W_{k}\right)_{k \geq 1}$ reflects the discharge timing and recruitment of the elementary signal sources (i.e., the motor units) involved; $H(z)$ describes the shape of the discharges (i.e., the action potentials) as well as the specific bioelectrical transfer function between generator and recording site. Measurement noise is neglected. $H(z)$ is modeled by an all-pole representation (autoregressive (AR) filter) of order $p$

$$
H(z)=\frac{1}{1+a_{1} z^{-1}+a_{2} z^{-2}+\cdots+a_{p} z^{-p}},
$$

where $a_{1}, a_{2}, \ldots, a_{p}$ are the AR coefficients and $z$ denotes the complex frequency of the $z$-transform. The time domain representation of this signal model is

$$
x_{k}=-\sum_{i=1}^{p} a_{i} x_{k-i}+w_{k} .
$$

As a particular feature of the signal model, the variance $\sigma^{2}(k)$ of the white noise excitation depends on the current state of the muscle activity which is modulated by descending commands from supraspinal levels as well as by feedback from peripheral receptors [16]. Within this framework, the indication for the response onset to be detected is an abrupt change in the variance profile $\sigma^{2}(k)$, that is, it changes abruptly from a pattern $\sigma_{0}^{2}(k)$ to a new pattern $\sigma_{1}^{2}\left(k, t_{0}\right) \neq \sigma_{0}^{2}(k)$ at time instant $t_{0}$.

Computerized onset detection requires to determine the unknown change time $t_{0}$ as accurate as possible. Most detection algorithms consist of up to three basic processing stages (see Figure 1):

- signal conditioning,

- detection unit,

- post-processor.

In an initial step, the observed SEMG signal passes through a signal conditioning unit in order to enhance the spectral content of the measured signal carrying information about the onset of muscle activation. Frequently, a lowpass filter is applied to reduce high frequency noise. More sophisticatedly, an adaptive whitening filter can be used which eliminates the spectral color that was introduced by the bioelectrical channel $H(z)$ but which is absolutely irrelevant for detection of changes in the excitation signal. The observed SEMG signal $\left(x_{k}\right)_{k \geq 1}$ is processed by a filter with transfer function

$$
H_{w}(z)=1+b_{1} z^{-1}+b_{2} z^{-2}+\cdots+b_{q} z^{-q}
$$

with the filter order $q$ and the filter coefficients $b_{i}$ tuned to the parameters of the shaping filter $H(z)$. Obviously, for $q=p$ and $b_{i}=a_{i}$, the whitening filter represents the ideal inverse filter $H_{w}(z)=H^{-1}(z)$ with respect to the transfer function $H(z)$. Therefore, with the parameters of $H(z)$ exactly known, the excitation $\left(w_{k}\right)_{k \geq 1}$ can completely be reconstructed. Usually, these parameters are unknown but can be estimated from the measured signal by some least-squares technique, for example [17].

In the next processing stage, a test function $g\left(y_{1}\right.$, $\left.y_{2}, \ldots, y_{k}\right)$ is computed from the (pre-conditioned) signal $\left(y_{k}\right)_{k \geq 1}$ which serves as an indicator for the response onset. The test function uses some or even all of the past samples to create an intermediate signal which is monitored by the decision rule in order to determine whether a change in the muscle activation pattern has occurred (alarm time $t_{\mathrm{a}}$ ). Some onset detection methods directly use $t_{\mathrm{a}}$ as an estimate $\hat{t}_{0}$ for the SEMG onset time $t_{0}$. Some other methods use $t_{\mathrm{a}}$ as a pure indicator for the existence of an onset and estimate $\hat{t}_{0}$ through additional postprocessing.

The decision rules of all methods will be written in stopping rule notation,

$$
t_{\mathrm{a}}=\min \left\{k \geq 1: g\left(y_{1}, y_{2}, \ldots, y_{k}\right) \geq h\right\},
$$




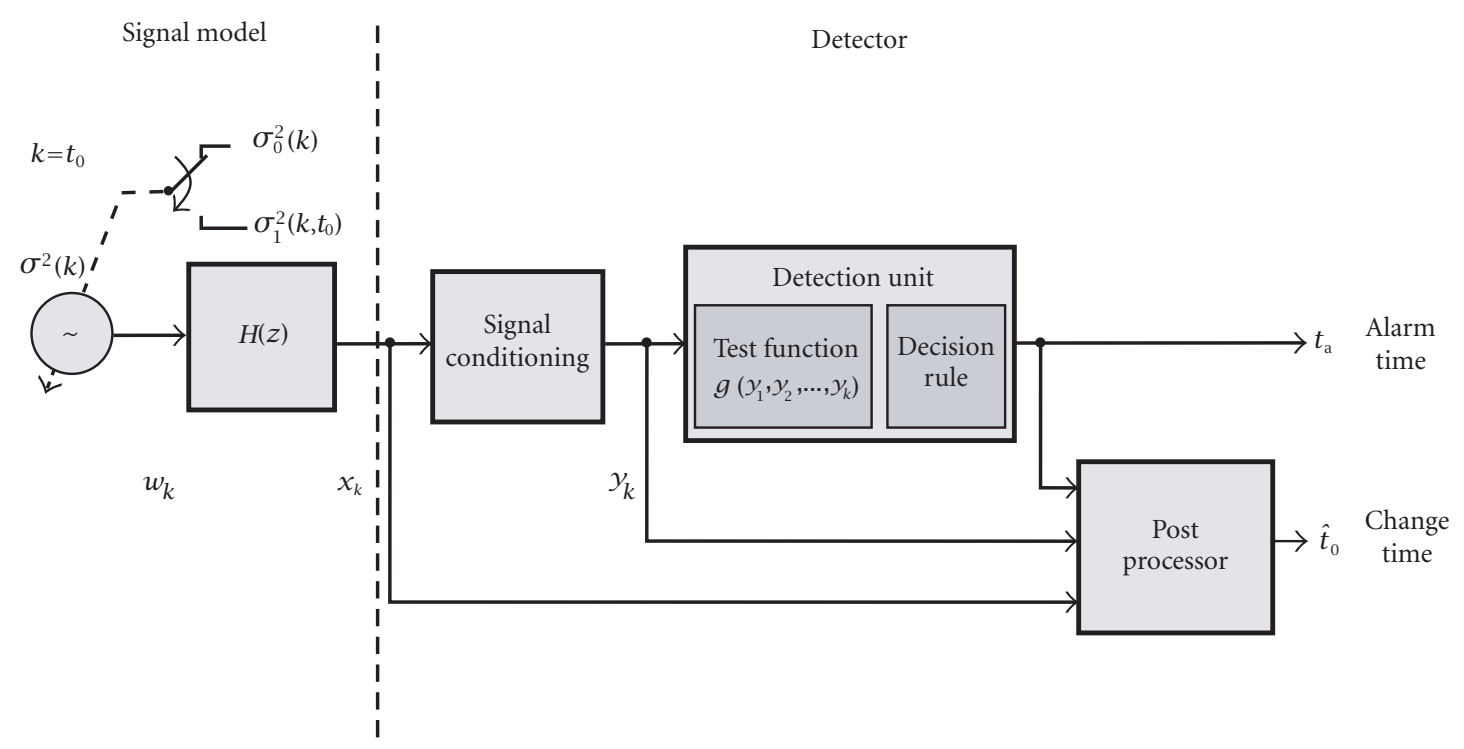

FIGURE 1: Scheme of event detection in surface electromyographic signal (SEMG). The digitized SEMG $x_{k}$ is modeled by a white Gaussian noise signal with dynamic variance $\sigma^{2}(k)$ exciting a linear system with transfer function $H(z)$. At $t_{0}$, the signal variance changes from resting activity $\sigma_{0}^{2}(k)$ to $\sigma_{1}^{2}\left(k, t_{0}\right)$ of the active state. Most detectors employ up to three processing stages (signal conditioning, detection unit, and post-processor) in order to detect an event (alarm time $t_{\mathrm{a}}$ ) and to determine an estimate for the unknown change time $t_{0}$.

where $h$ is an appropriate threshold. Each time a new data point $y_{k}$ is available, the current value $g_{k}$ of the test function is computed from the samples $y_{1}, y_{2}, \ldots, y_{k}$ and compared to the threshold $h$. As long as $g_{k}<h$, this procedure is repeated sample by sample. At the first time instant $t_{\mathrm{a}}$, when $g_{k} \geq h$, the procedure is stopped, and an event alarm is given.

Estimation of the exact change time $t_{0}$ by the postprocessor usually starts after event alarm was given at $t_{\mathrm{a}}$, and the estimate $\hat{t}_{0}$ of the unknown $t_{0}$ is computed from the samples $y_{1}, y_{2}, \ldots, y_{t_{\mathrm{a}}+\Delta}$. Some methods employ a separate signal conditioning unit which computes the input sequence for the post-processor from the raw SEMG $x_{1}, x_{2}, \ldots, x_{t_{\mathrm{a}}+\Delta}$. Most algorithms require an additional amount $\Delta$ of samples after the alarm time, thus $t_{\mathrm{a}}+\Delta$ represents the earliest time instant when the final onset estimate $\hat{t}_{0}$ is available. The (optional) change time estimation procedure is written as

$$
\hat{t}_{0}=f\left(y_{1}, y_{2}, \ldots, y_{t_{\mathrm{a}}+\Delta}\right) \text {, }
$$

where $f$ is an arbitrary function that computes the change time from $y_{1}, y_{2}, \ldots, y_{t_{\mathrm{a}}+\Delta}$.

In the following two sections, commonly used detection methods are described within the framework of this basic computational structure shown in Figure 1. The summary of this analysis is presented in Table 1, together with the parameter values chosen for their performance evaluation.

\subsection{Selected threshold-based onset detection methods}

\section{Hodges and Bui}

The detection algorithm proposed by Hodges and Bui [13] is a representative for a large class of detection methods, known as finite moving average (FMA) algorithms (cf. [5, 8, 18, 19]). All these methods employ a fixed-size sliding test window, and the output at time $k$ is computed as the weighted sum of the $W$ signal samples $y_{k-W+1}, y_{k-W+2}, \ldots, y_{k}$ contained within the window. Within the framework of Figure 1, the Hodges method first conducts some signal conditioning; SEMG data samples are rectified and subsequently lowpass filtered. From the pre-processed signal $\left(y_{k}\right)_{k \geq 1}$, the test function $g\left(y_{1}, y_{2}, \ldots, y_{k}\right)$ at time $k$ is computed as the mean of $y_{k-W+1}, y_{k-W+2}, \ldots, y_{k}$, and muscle activity onset is identified at the instant when the test function exceeds the baseline activity level by a specified multiple $h$ of standard deviations. The baseline activity is adaptively determined by averaging the initial $M$ samples of $\left(y_{k}\right)_{k \geq 1}$. The algorithm can be summarized by the following decision rule:

$$
\begin{aligned}
t_{\mathrm{a}} & =\min \left\{k \geq W: g_{k} \geq h\right\}, \\
g_{k} & =\frac{1}{\hat{\sigma}_{0}}\left(\tilde{y}_{k}-\hat{\mu}_{0}\right), \\
\tilde{y}_{k} & =\frac{1}{W} \sum_{i=k-W+1}^{k} y_{k}, \\
\hat{t}_{0} & =t_{\mathrm{a}}-W+1,
\end{aligned}
$$

where $y_{k}$ denotes the rectified and lowpass filtered SEMG signal, and $\hat{\mu}_{0}$ and $\hat{\sigma}_{0}$ are the mean and standard deviation of the $M$ initial samples of $\left(y_{k}\right)_{k \geq 1}$, respectively. After the stopping rule signaled an alarm, the first index of the sliding window is used as an estimate for the onset time $t_{0}$, which is a very simple post-processor. The Hodges method was implemented with the following parameters: $h=2.5$, sixth-order digital butterworth lowpass filter with $50 \mathrm{~Hz}$ cutoff frequency, $W=50, M=200$. 
TABLE 1: Characteristic properties of all examined onset detection methods.

\begin{tabular}{|c|c|c|c|c|c|c|c|}
\hline & $\begin{array}{l}\mathscr{3} \\
\mathbb{8} \\
\mathbb{1}\end{array}$ & 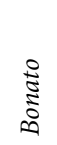 & 苞 & 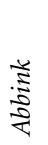 & 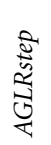 & & 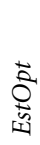 \\
\hline \multicolumn{8}{|l|}{ Signal conditioning } \\
\hline Pre-whitening & & $x$ & & & $x$ & $x$ & $x$ \\
\hline Data sample rectification & $\times$ & & $\times$ & $\times$ & & & \\
\hline Data sample squaring & & $\times$ & & & $\times$ & $x$ & $x$ \\
\hline Lowpass filtering & $\times$ & & & $x$ & & & \\
\hline \multicolumn{8}{|l|}{ Test function/decision rule } \\
\hline Moving average + simple threshold & $\times$ & & $\times$ & $x$ & & & \\
\hline$\chi^{2}$ test variable + double threshold & & $\times$ & & & & & \\
\hline Likelihood ratio test & & & & & $x$ & $\times$ & $x$ \\
\hline \multicolumn{8}{|l|}{ Post-processor/onset time estimation } \\
\hline Any threshold crossing & $\times$ & & & & & & \\
\hline Threshold \& duration-based selection of alarm time & & $x$ & $\times$ & & & & \\
\hline Search for maximum in (additional) test function & & & & $\times$ & $x$ & $x$ & $x$ \\
\hline
\end{tabular}

\section{Bonato et al.}

The signal conditioning stage of the Bonato method [14] consists of an adaptive whitening filter, with the filter parameters estimated from the current SEMG data. The test function is determined by two successive samples of $\left(y_{k}\right)_{k \geq 1}$ according to

$$
g_{k}=\frac{1}{\hat{\sigma}_{0}^{2}}\left(y_{k-1}^{2}+y_{k}^{2}\right),
$$

where $\hat{\sigma}_{0}$ is the standard deviation of the $M$ initial samples of $\left(y_{k}\right)_{k \geq 1}$. Note that $g_{k}$ is evaluated only for odd values of $k$. The decision rule is

$$
t_{\mathrm{a}}=\min \left\{k=1,3,5, \ldots: g_{k} \geq h\right\}
$$

and the post-processor checks the resulting alarms for their relevance. It accepts the onset decision only, if both following rules apply:

- at least $n$ out of $m$ successive samples must exceed the threshold in order to indicate the current muscle state to be an active state,

- such an active state must last for at least $T_{1}$ samples.

If this check holds true, the earliest beginning of this active state epoch is taken as an estimate for the onset time $t_{0}$.

As an important property, the method assumes that the pre-whitened SEMG $\left(y_{k}\right)_{k \geq 1}$ before the response onset is represented by a sequence of statistically independent zero mean Gaussian random variables with equal variance. Thus, with $\hat{\sigma}_{0}^{2}$ estimated from the measured sequence, the statistical properties of the test function $g_{k}$ can be explicitly specified, which facilitates a more sophisticated setting of the parameters $h, n, m$, and $T_{1}$. Further, the use of a double-threshold scheme in the post-processor provides a higher degree of freedom for selecting detection parameters. However, due to the implicit maximum operation of the " $n$ out of $m$ " criterion the accuracy with which the response onset can be determined is limited by the size $m$ of the test window. Moreover, the down-sampling operation reduces time resolution which may also lead to a less accurate onset estimation. Bonato was implemented with the parameters $h=7.74, n=1$, $m=5, T_{1}=50$, and $M=200$.

\section{Lidierth}

Lidierth [15] proposed a full-wave rectification of the raw SEMG as signal conditioning only. The test function and decision rule of the Lidierth detection unit are identical to the Hodges approach, but increased performance is achieved by the extended post-processing rules:

- SEMG onset detection is accepted, if the test function exceeds the threshold $h$ to the active level for at least $T_{1}$ samples, and

- during $T_{1}$, the test function may repeatedly fall below the threshold, but each time not longer than $T_{2}$ samples.

Note that these post-processing rules represent a specific case of the post-processor suggested by Bonato; with the parameters of the Bonato " $n$ out of $m$ " criterion set to $n=1$ and $m=T_{2}$, both post-processing rules are identical, thus Lidierth is a composite of Bonato and Hodges. Lidierth was implemented with parameters $T_{1}=90, T_{2}=15, h=3$, and $M=200$.

\section{Abbink et al.}

Again, the "new" method copies the signal conditioning and detection unit from already known methods, and adds some specific fine tuning by extending the postprocessor function: Abbink [2] uses the Hodges signal conditioning and detection unit approach, only changing the cutoff frequency to $3 \mathrm{~Hz}$ and the window length $W$ to 1 .

The post-processor stage of Abbink uses a less smooth version $\left(y_{k}^{\prime}\right)_{k \geq 1}$ of SEMG, which is obtained by applying a but- 
terworth lowpass with $30 \mathrm{~Hz}$ cutoff frequency to the rectified SEMG (i.e., the post-processor does his own signal conditioning). Looking backwards from $t_{\mathrm{a}}$, the following rules are applied:

$$
\begin{aligned}
\hat{t}_{0} & =\arg \max _{N \leq j \leq t_{\mathrm{a}}}\left\{n_{\text {low }}(j)+n_{\text {high }}(j)\right\}, \\
n_{\text {low }}(j) & =\sum_{i=j-N+1}^{j} \operatorname{Ind}\left\{\frac{1}{\hat{\sigma}_{0}}\left(y_{i}^{\prime}-\hat{\mu}_{0}\right)<h_{2}\right\}, \\
n_{\text {high }}(j) & =\sum_{i=j+1}^{j+N} \operatorname{Ind}\left\{\frac{1}{\hat{\sigma}_{0}}\left(y_{i}^{\prime}-\hat{\mu}_{0}\right)>h_{2}\right\}, \\
\operatorname{Ind}(x) & = \begin{cases}1 & \text { if } x \text { is true, } \\
0 & \text { otherwise, }\end{cases}
\end{aligned}
$$

where $n_{\text {low }}(j)$ denotes the number of normalized amplitudes smaller than the threshold $h_{2}$ in the $N$ samples directly preceding the onset time candidate $j$, and $n_{\text {high }}(j)$ is the number of amplitudes larger than $h_{2}$ in the $N$ samples directly following $j$. Mean $\hat{\mu}_{0}$ and standard deviation $\hat{\sigma}_{0}$ are estimated from the initial $M$ samples of $\left(y_{k}^{\prime}\right)_{k \geq 1}$, respectively. The following parameters were used for the simulations: $h=3, h_{2}=3$, $N=200$, and $M=200$.

\subsection{Detectors based upon statistically optimal decision}

If the signal generating process is (partially) known, detectors based on statistically optimal decision rules can be used. In this section, three algorithms are presented which are derived from the model-based detection concept of [12]. In order to determine an estimate of $t_{0}$, these methods evaluate the statistical properties of the measured SEMG signal before and after a possible change in model parameters. Only basic processing steps are described here (see [12] for details). The specific implementations of the decision rules for the SEMG signal model (see Figure 1 left) are given in the appendix.

For signal conditioning, all 3 methods employ an adaptive whitening filter for the reduction of irrelevant information. Assuming optimal performance of the filter, the (reconstructed) excitation signal $\left(y_{k}\right)_{k \geq 1}=\left(w_{k}\right)_{k \geq 1}$ represents a single realization of a statistically independent Gaussian random process $\left(Y_{k}\right)_{k \geq 1}$. The statistical properties of an individual random variable $Y_{k}$ can be fully described by its probability density function (PDF)

$$
p_{\sigma}\left(y_{k}\right)=\frac{1}{\sqrt{2 \pi \sigma^{2}(k)}} e^{-y_{k}^{2} / 2 \sigma^{2}(k)},
$$

which depends upon the (deterministic) variance profile $\sigma^{2}(k)$. The model-based decision rules for this problem depend on the a priori knowledge about the variance profiles $\sigma_{0}^{2}(k)$ and $\sigma_{1}^{2}\left(k, t_{0}\right)$ before and after the change to be detected.

\section{Optimal estimator (EstOpt)}

If the variance profiles $\sigma_{0}^{2}(k)$ and $\sigma_{1}^{2}\left(k, t_{0}\right)$ are exactly known (except the onset time $t_{0}$ ), a statistically optimal decision rule can be designed according to

$$
\begin{aligned}
t_{\mathrm{a}} & =\min \left\{k \geq 1: g_{k} \geq h\right\}, \\
g_{k} & =\max _{1 \leq j \leq k} S_{j}^{k}, \\
S_{j}^{k} & =\frac{1}{2} \sum_{i=j}^{k}\left[\left(\sigma_{0}^{-2}(i)-\sigma_{1}^{-2}(i, j)\right) y_{i}^{2}+\ln \frac{\sigma_{0}^{2}(i)}{\sigma_{1}^{2}(i, j)}\right], \\
\hat{t}_{0} & =\arg \max _{1 \leq j \leq t_{\mathrm{a}}} S_{j}^{t_{\mathrm{a}}} .
\end{aligned}
$$

This detector referred to as EstOpt algorithm computes the maximum likelihood (ML) estimate $\hat{t}_{0}$ of the unknown onset time $t_{0}$ provided that all remaining parameters of the signal model are exactly known. The EstOpt decision rule is suited for arbitrary but known dynamic variance profiles $\sigma_{0}^{2}(k)$ and $\sigma_{1}^{2}(k, j)$, thus it shows optimal performance among all possible algorithms for sequential detection in this application. Therefore, the EstOpt detector may serve as a reference for performance rating. (Essentially, the test compares the loglikelihood ratio

$$
S_{j}^{k}=\sum_{i=j}^{k} \ln \frac{p_{\sigma_{1}}\left(y_{i}, j\right)}{p_{\sigma_{0}}\left(y_{i}\right)}
$$

between the two distributions before and after a possible change at time $j$ with a threshold $h$. Since the exact change time $t_{0}$ is unknown, it is replaced by its ML estimate, that is, a maximum operator selects the largest value of the test function with respect to all possible change times $1 \leq j \leq k$. Each time a new data point $y_{k}$ is available, a test window with the upper bound $k$ fixed at the current observation and with its lower bound $j$ comprises all the observations $y_{j}, y_{j+1}, \ldots, y_{k}$ after the hypothetical change time $j$. For each candidate $j$, the $\log$-likelihood ratio $S_{j}^{k}$ is computed from the observations within the test window. If the maximum of $S_{j}^{k}$ with respect to all hypothetical change times $1 \leq j \leq k$ exceeds the threshold $h$, data acquisition stops and event alarm is given (alarm time $t_{\mathrm{a}}$ ). The time $j$ at which the maximum value is obtained serves as the maximum likelihood estimate $\hat{t}_{0}$ of the unknown change time $t_{0}$.)

\section{Approximated generalized likelihood-ratio detectors (AGLRstep, AGLRramp)}

If the variance profiles $\sigma_{0}^{2}(k)$ and $\sigma_{1}^{2}(k, j)$ are not known like in $E s t O p t$, they can be replaced by estimates. For this condition, the approximated generalized likelihood ratio (AGLR) detector [20] is an appropriate tool to formulate the stopping rule. First, the variance profiles are assumed to depend upon several unknown parameters, that is,

$$
\begin{aligned}
\sigma_{0}^{2}(k) & =\sigma_{0}^{2}\left(k, \theta_{0}\right), \\
\sigma_{1}^{2}(k, j) & =\sigma_{1}^{2}\left(k, j, \theta_{1}\right) .
\end{aligned}
$$

The unknown parameter vector $\theta_{0}$, true up to $t_{0}$, is estimated from the first $M$ observations of the SEMG by ML techniques, resulting in 


$$
\hat{\theta}_{0}=\arg \sup _{\theta_{0}}\left(\sum_{i=1}^{M} \ln p_{\sigma_{0}}\left(y_{i}, \theta_{0}\right)\right),
$$

which is kept fixed throughout the remaining detection procedure. Next, a sliding window of fixed size $W$ is continuously shifted along the data sequence. For each location $k$ of the window, the ML estimate $\hat{\theta}_{1}$ of the unknown parameter vector $\theta_{1}$ after change is determined from the $W$ data points covered by the window, and the corresponding log-likelihood ratio $\hat{S}_{k-W+1}^{k}$ is computed and compared with a threshold $h$. Finally, after a change has been indicated, the exact change time is estimated by an ML procedure from all possible candidates $j \leq t_{\mathrm{a}}$. The AGLR decision rule can be summarized as

$$
\begin{aligned}
& t_{\mathrm{a}}=\min \left\{k \geq W: g_{k} \geq h\right\}, \\
& g_{k}=\hat{S}_{k-W+1}^{k}, \\
& \hat{S}_{j}^{k}=\sup _{\theta_{1}} \frac{1}{2} \sum_{i=j}^{k}[\left(\sigma_{0}^{-2}\left(i, \hat{\theta}_{0}\right)-\sigma_{1}^{-2}\left(i, j, \theta_{1}\right)\right) y_{i}^{2} \\
&\left.\quad+\ln \frac{\sigma_{0}^{2}\left(i, \hat{\theta}_{0}\right)}{\sigma_{1}^{2}\left(i, j, \theta_{1}\right)}\right], \\
& \hat{t}_{0}=\arg \max _{W \leq j \leq t_{\mathrm{a}}}\left(\hat{S}_{j}^{t_{\mathrm{a}}+\Delta}\right),
\end{aligned}
$$

where $\Delta$ is an appropriate dead zone which ensures that a minimum number of observations is available for parameter estimation. Note that the maximization with respect to $\theta_{1}$ has to be repeatedly performed for each combination $(j, k)$ to be tested. Therefore, the computational effort is dominated by the number of operations required for computing $\hat{\theta}_{1}$.

In this paper, two implementations of the AGLR decision rule are used which differ in the complexity of $\sigma_{1}^{2}(k, j)$. The AGLRstep algorithm assumes a step-like variance profile with constant but different variances before and after change. This allows for a very efficient implementation of the method but completely disregards the available information about the dynamic profile of the change itself. The AGLRramp algorithm explicitly takes this information into account by assuming a "ramp and hold" change profile. The unknown parameters $\theta_{1}$ of the transition are estimated from the actual observations. Thus, comparison of the AGLRstep and AGLRramp methods shows the advantage of using available information about the change dynamics in the detection process. Their exact implementations for the present signal model are given in the appendix.

\section{EVALUATION OF DETECTION PERFORMANCE ON SIMULATED SEMG RECORDINGS}

\subsection{Signal generation}

All methods were tested for robustness and efficiency on a set of simulated SEMG traces which were generated by using the signal model shown in Figure 1(left). The coefficients of the shaping filter were determined from a representative set of real SEMG data from a previous study [21] using standard techniques of least squares parameter estimation [17]. Model order was set to $p=8$. A total number of 16000 segments, each consisting of 1000 data points (i.e., $1000 \mathrm{~ms}$ record length) were generated. Figure 2 depicts the principle and definitions. The response to be detected was modeled by a rapid change in the variance $\sigma^{2}(k)$ from a pattern

$$
\sigma_{0}^{2}(k)=\sigma_{\text {noise }}^{2}
$$

to a new pattern

$$
\sigma_{1}^{2}\left(k, t_{0}\right)=\sigma_{\text {noise }}^{2}+\sigma_{\text {signal }}^{2} u\left(k, t_{0}\right),
$$

where $t_{0}$ denotes the onset of the voluntary muscle activation, $u\left(k, t_{0}\right)$ describes the dynamic profile of the change, and $\sigma_{\text {noise }}^{2}$ and $\sigma_{\text {signal }}^{2}$ are appropriate constants. The resting activity $\sigma_{\text {noise }}^{2}>0$ results from spontaneous firing of motor units which occurs even if the muscle is not voluntarily activated. (Note that the usage of the term "noise" is technically but not biologically motivated.) $\sigma_{\text {signal }}^{2}$ denotes the magnitude of the activation pattern. The dynamic change profile was approximated by a unit ramp and hold transition

$$
u\left(k, t_{0}\right)= \begin{cases}0 & \text { when } k \prec t_{0}, \\ \frac{\left(k-t_{0}\right)}{\tau} & \text { when } t_{0} \leq k \leq t_{0}+\tau, \\ 1 & \text { when } k \succ t_{0}+\tau,\end{cases}
$$

where $\tau$ denotes the duration of the ramp. This finite slope of the change profile reflects the limited dynamics within the neuromuscular system [16], and $\tau$ was varied as it can be observed in real data. The onset $t_{0}$ of voluntary muscle activation was randomly varied between $400 \mathrm{~ms}$ and $600 \mathrm{~ms}$, relative to the beginning of the trace. Change magnitude $\sigma_{\text {signal }}^{2}$ was always set to 1 while background activity $\sigma_{\text {noise }}^{2}$ was varied to create variable signal-to-noise ratios (SNR)

$$
\mathrm{SNR}=10 \cdot \log _{10} \frac{\sigma_{\text {signal }}^{2}}{\sigma_{\text {noise }}^{2}}[\mathrm{~dB}] .
$$

Several sets of simulated SEMG traces, each consisting of 4000 trials, were used for testing the onset detection algorithms. The assessment of the onset detection accuracy was made for the following range of parameters (referenced as Mixed Trials):

- ramp duration $\tau$ uniformly distributed between $5 \mathrm{~ms}$ and $30 \mathrm{~ms}$,

- signal-to-noise ratio SNR uniformly distributed between $6 \mathrm{~dB}$ and $12 \mathrm{~dB}$.

In particular, dependence on SNR and ramp duration were studied. Sensitivity to SNR was investigated with the Mixed SNR Trials data set:

- fixed ramp duration $\tau$ of 20 samples,

- SNR uniformly distributed between $6 \mathrm{~dB}$ and $12 \mathrm{~dB}$. Performance for extremely small SNR was evaluated with the Fixed SNR Trials data set: 


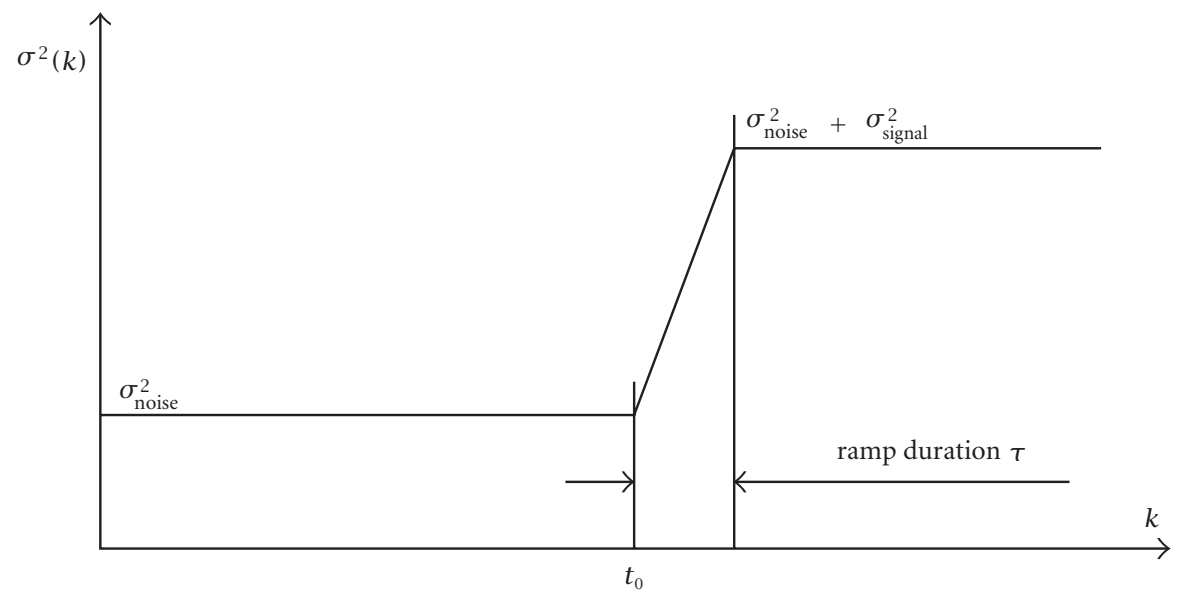

(a) Power envelope of modeled SEMG signals. After a period of background activity the SEMG power linearly rises to the active state within $\tau$. Parameters were systematically varied during simulation.

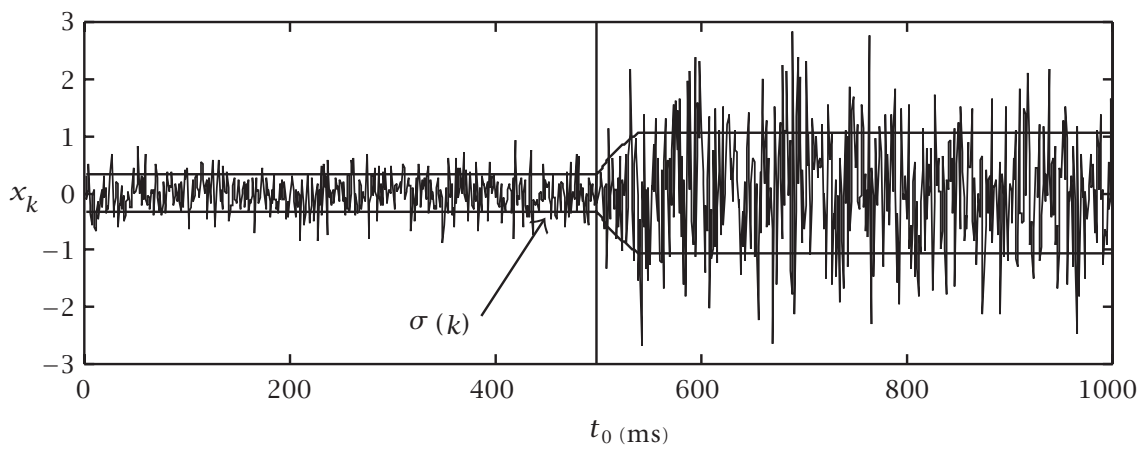

(b) Sample trace of simulated SEMG.

FIGURE 2: Simulated SEMG.

- fixed ramp duration $\tau$ of 20 samples,

- fixed SNR of either $3 \mathrm{~dB}$ or $6 \mathrm{~dB}$.

Sensitivity to ramp duration was investigated with the Mixed Ramp Trials data set:

- ramp duration $\tau$ uniformly distributed between $5 \mathrm{~ms}$ and $30 \mathrm{~ms}$,

- fixed SNR of $10 \mathrm{~dB}$.

All simulations were performed using MATLAB $^{\circledR}$ (version 5.3, The MathWorks, Natick, MA) on an IBM-compatible PC.

\subsection{Results of onset detection analysis}

The aim of this paper is to objectively compare the performance of commonly used onset detection methods. It should be considered that several factors could influence detection performance:

- algorithmic delay of onset detection,

- percentage of missed responses and false alarm rate,

- detection error bias, that is, average deviation between estimated and true onset,

- reproducibility for signals with the same parameters,
- sensitivity of all attributes to SNR and onset ramp duration.

The first two factors refer to the detection power of the algorithm, that is, the ability of a method to signal an event as early as possible (small delay of alarm time $t_{\mathrm{a}}$ ) combined with a false alarm rate as low as possible. Actually, due to the relatively high SNR, the majority of the simulated SEMG responses could be detected within a reasonable time range around the real onset, and the detection probability of all algorithms was nearly $100 \%$. We, therefore, focus on the remaining 3 factors which are related to the accuracy of the estimated change times $t_{0}$.

\section{Measures of detection performance}

Figure 3 shows the probability density functions (PDFs) of the onset error $\varepsilon=\hat{t}_{0}-t_{0}$ for all methods (Mixed Trials). Two diagrams with different abscissa scales are used for improved visibility. The optimal estimator EstOpt indicating the upper performance limit provides a rather narrow distribution which is almost symmetrical to the origin. The AGLRramp and AGLRstep methods are showing increasing asymmetry and width of the distributions. While the PDF of the 


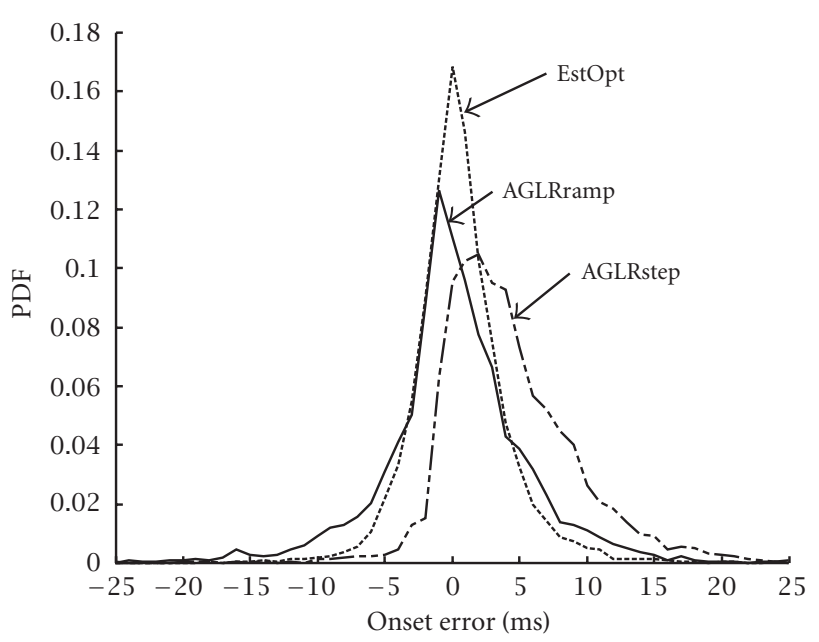

(a)

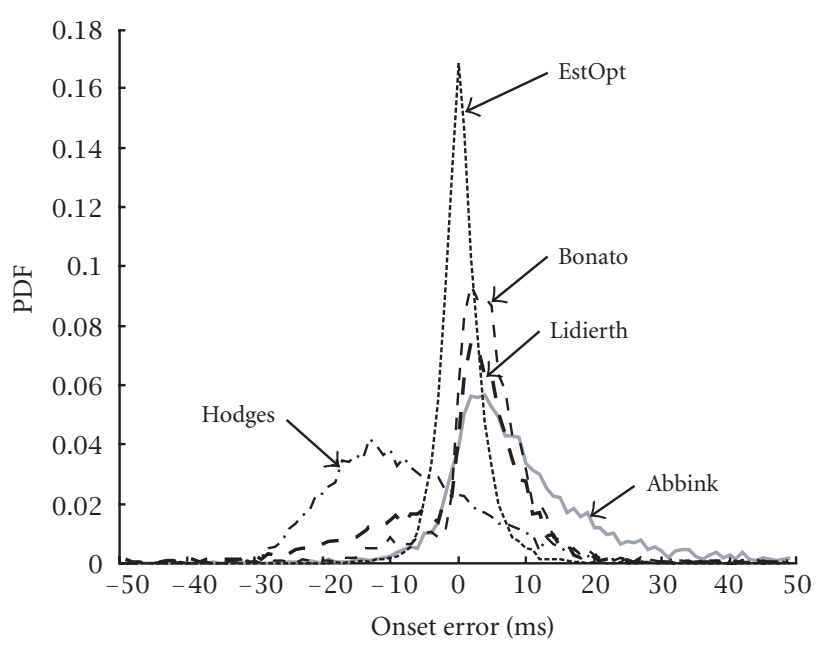

(b)

Figure 3: Probability density functions (PDFs) of onset estimation errors for the Mixed Trials data set (SNR 6-12 dB, ramp duration 5-30 ms). Note the different abscissa scaling in (a) and (b).

AGLRramp method is still centered near the origin, the PDF of AGLRstep is shifted towards higher onset errors. In the performance ranking, Bonato and Lidierth follow with reasonable results. Finally, Abbink shows worse results because the PDF spreads to larger onset error values, and Hodges ranks lowest because of a wide-spread distribution with additionally strong (negative) bias.

Table 2 depicts mean and standard deviation of onset estimation errors and the percentage of detected onsets with an absolute error less than $100 \mathrm{~ms}$ for each method. The $100 \mathrm{~ms}$ limit was chosen because it comprises at least 99\% of all onset estimates computed from the Mixed SEMG Trials. Note that, due to the very thin tail of the PDF, variations in the limit will result in only minor variations of the resulting probability of detected onset. Analysis of the mean errors in Table 2 shows that the AGLRramp algorithm provides
TABLe 2: Statistics for onset estimates based on SEMG data set "Mixed Trials."

\begin{tabular}{lclr}
\hline Method & $\begin{array}{c}\text { Detected } \\
\text { onsets }\end{array}$ & $\begin{array}{l}\text { Mean } \\
\text { error }\end{array}$ & $\begin{array}{r}\text { Error } \\
\text { STD }\end{array}$ \\
\hline EstOpt & $100.0 \%$ & $0.6 \mathrm{~ms}$ & $3.6 \mathrm{~ms}$ \\
AGLRramp & $99.7 \%$ & $0.2 \mathrm{~ms}$ & $5.4 \mathrm{~ms}$ \\
AGLRstep & $99.8 \%$ & $4.2 \mathrm{~ms}$ & $5.0 \mathrm{~ms}$ \\
Bonato & $99.9 \%$ & $4.0 \mathrm{~ms}$ & $7.5 \mathrm{~ms}$ \\
Lidierth & $98.9 \%$ & $0.1 \mathrm{~ms}$ & $11.1 \mathrm{~ms}$ \\
Abbink & $99.6 \%$ & $8.8 \mathrm{~ms}$ & $10.4 \mathrm{~ms}$ \\
Hodges & $99.9 \%$ & $-7.1 \mathrm{~ms}$ & $11.8 \mathrm{~ms}$ \\
\hline
\end{tabular}

estimates very close to the "true" onset. AGLRstep, however, yields a positive bias of $4.2 \mathrm{~ms}$ reflecting the fact that the steplike change profile assumed by AGLRstep only represents an approximation for the more gradual "true" change profile. The improvement in bias obtained by using the more realistic change pattern of the AGLRramp algorithm, however, is at the expense of a slightly increased standard deviation. This is because estimation of the additional parameter introduces additional uncertainty to the final detection result.

The accuracy function

$$
P_{\mathrm{a}}=P\left(\left|\hat{t}_{0}-t_{0}\right| \leq a\right)
$$

shown in Figure 4 can be used as another measure of detection performance. The accuracy function denotes the percentage of responses that were detected with an absolute error smaller than or equal to a maximum tolerated error $a$ for different values of $a$. For large $a$, the diagram reflects the general ability of a method to detect an onset, whereas a particular accuracy requirement of a method can specifically be assessed at small values $a$. Thus, the more a curve approaches the upper left corner, the higher is the onset detection quality. As a major result, the accuracy functions in Figure 4 confirm the ranking already obtained from the PDFs shown in Figure 3.

\section{Effect of signal to noise ratio}

Figure 5 depicts the dependence of mean and standard deviation of the onset error on SNR (Mixed SNR Trials). All methods show a systematic degradation of detection performance for smaller SNR. Except the Hodges approach (sensitivity to SNR of approximately $1 \mathrm{~ms} / \mathrm{dB}$ ), all methods show a similar relative SNR sensitivity of the standard deviation of approx. $0.5 \mathrm{~ms} / \mathrm{dB}$. Generally, the overall variability is significantly smaller for the methods employing a whitening filter (EstOpt, AGLRstep, AGLRramp, Bonato) and decreases with increasing level of a priori knowledge used. Again, the AGLRramp provides the smallest bias among all detectors showing a zero mean estimation error close to the EstOpt reference (see Figure 5b). Most importantly, the AGLRramp detector is the only approach which is nearly insensitive to SNR. All other methods show biased results with an SNR sensitivity of $0.3-0.8 \mathrm{~ms} / \mathrm{dB}$. The poor results of the Hodges approach (sensitivity $3.3 \mathrm{~ms} / \mathrm{dB}$ ) demonstrate the general problem of any simple adaptive threshold detector; the threshold level is 


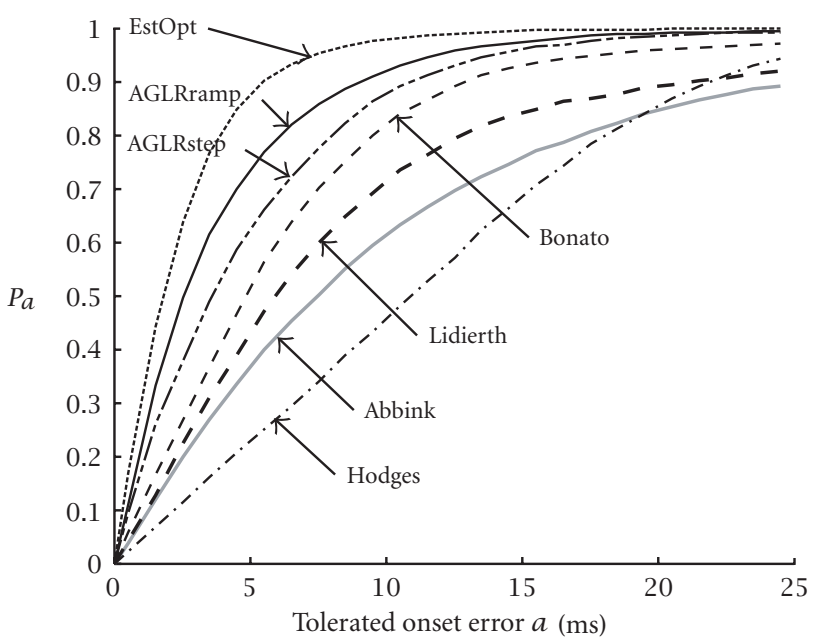

Figure 4: Accuracy functions of onset estimates (Mixed Trials data set). The ordinate value denotes the probability that the tolerated error $a$ given by the abscissa value is not exceeded. The further a curve is bent to the upper-left corner, the better is the onset detection quality.

usually adapted to the background activity (noise) and thus varies with SNR. The higher the noise level (decreasing SNR), the larger the threshold and, consequently, the later the onset will be detected.

Degradation of performance with smaller SNR is particularly obvious from the accuracy functions in Figure 6 (Fixed $S N R$ Trials). For SNR $=6 \mathrm{~dB}$, all methods provided acceptable results (see Figure 6a) but decreasing signal quality to $\mathrm{SNR}=3 \mathrm{~dB}$ promptly reveals their specific quality and robustness (see Figure 6b). The optimal reference EstOpt still detects 93\% (SNR $6 \mathrm{~dB}$ ) and $82 \%$ (SNR $3 \mathrm{~dB}$ ) of all onsets with an accuracy of $10 \mathrm{~ms}$. AGLRramp and AGLRstep follow closely with a near-zero rate of undetected onsets. Even for the $3 \mathrm{~dB}$ trials, more than $98 \%$ of onsets are detected with an absolute error less than $50 \mathrm{~ms}$. But all the purely threshold-based methods (Abbink, Bonato, Lidierth, Hodges) cannot compete with likelihood-based methods; especially for the $3 \mathrm{~dB}$ situation, their performance deteriorates extremely.

\section{Effect of change dynamics}

Figure 7 illustrates the dependence of mean onset error on ramp duration $\tau$ (Mixed Ramp Trials). AGLRramp is designed to compensate change dynamics, and, thus results are closest to those of EstOpt. But Bonato, Lidierth, and AGLRstep show a similar small sensitivity to ramp duration, only Abbink and Hodges are rather sensitive. Variability of onset estimates (around mean onset error) was not affected by variations in ramp duration.

Generally, changing SNR predominately affects the onset error PDF width and the percentage of undetected (missed) onsets, whereas increasing ramp duration leads to a delayed onset detection (onset error bias).

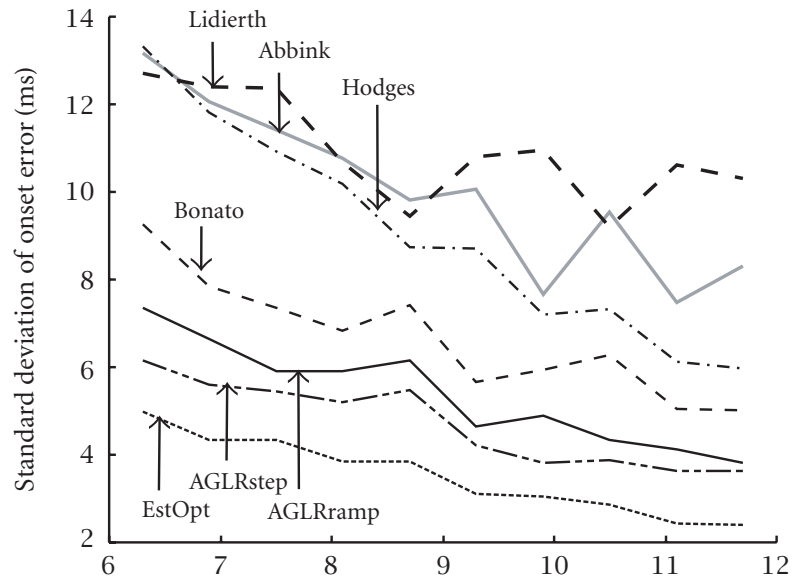

(a) Standard deviation of onset error.

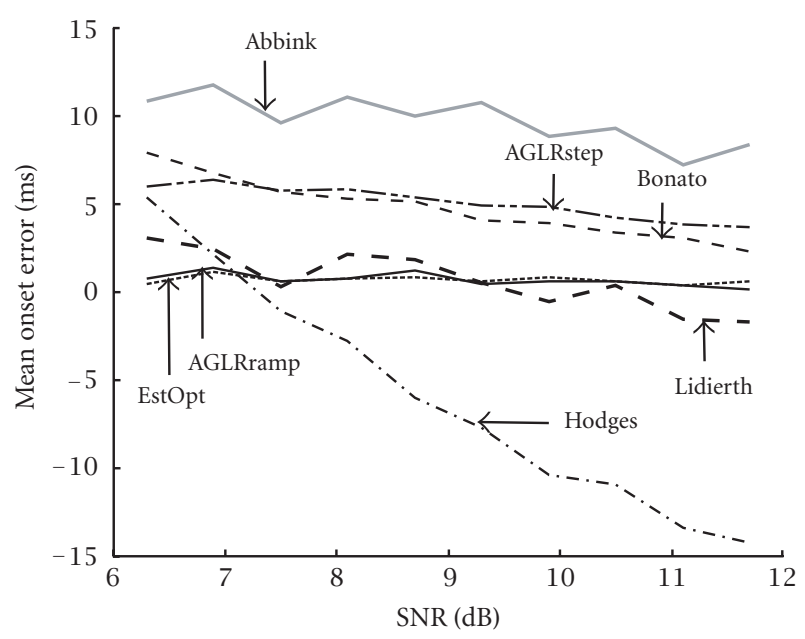

(b) Mean onset error.

FIGURE 5: Dependence of onset estimation error on signal-to-noise ratio (SNR) for the Mixed SNR Trials data set (SNR 6-12 dB, ramp duration $20 \mathrm{~ms}$ ).

\section{EVALUATION OF DETECTION PERFORMANCE ON REAL SEMG RECORDINGS}

\subsection{Data acquisition}

All methods were additionally tested on real SEMG records obtained in a reaction time situation. Briefly, five subjects without any sign of neurological deficits were examined. They performed rapid stimulus initiated transient lateral abductions of the index finger in response to a bright visual "go" signal displayed at random times. During the experiment, the tip of the index finger was fixed to a lever which restricted movement to the horizontal plane. SEMG activity of the first dorsal interosseus (FDI) muscle was recorded using an active surface electrode (Liberty Mutual MYO 111). SEMG signals were digitized for a period of $6 \mathrm{~s}$ around the stimulus onset with a sampling rate of 1000/s. After short practice, each sub- 


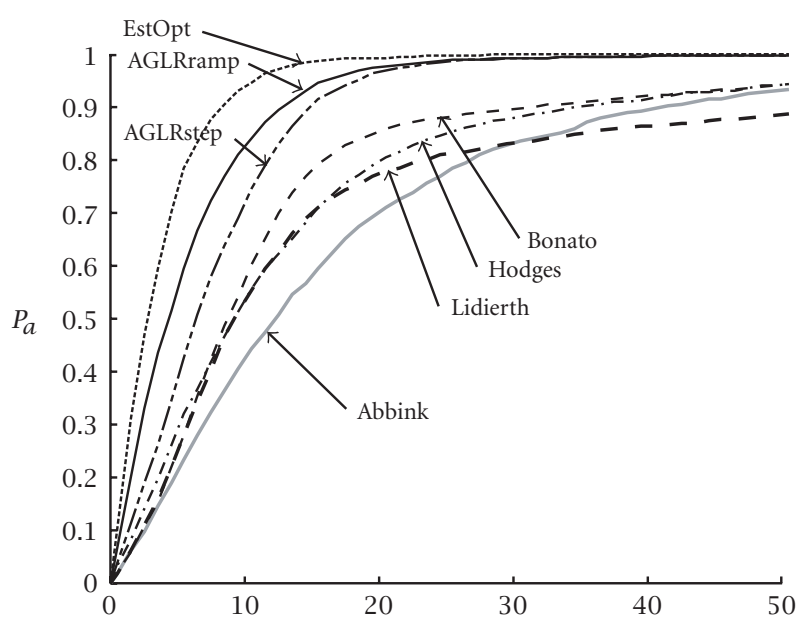

(a) $\mathrm{SNR}=6 \mathrm{~dB}$.

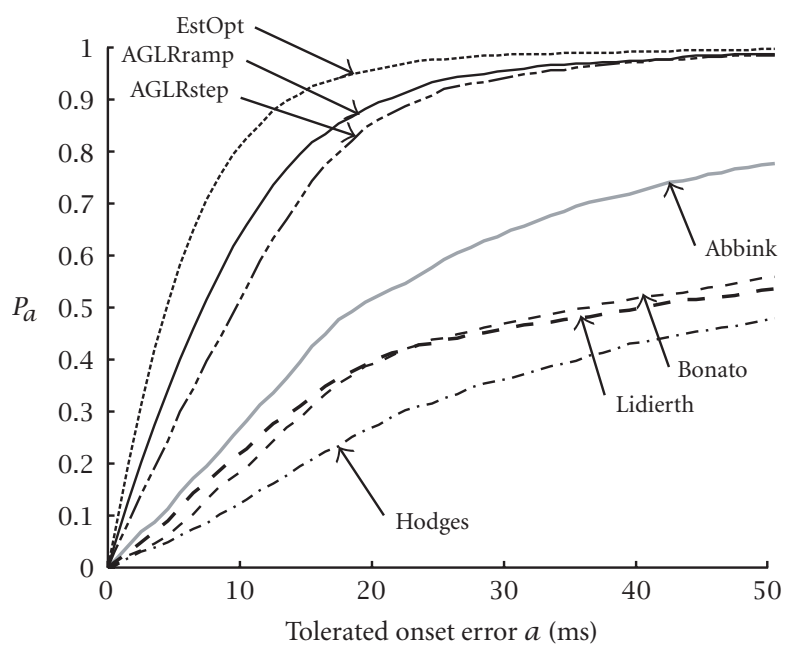

(b) $\mathrm{SNR}=3 \mathrm{~dB}$.

FIGURE 6: Dependence of accuracy functions on SNR for constant ramp duration of $20 \mathrm{~ms}$.

ject produced 120 single responses. The recorded data were visually inspected and only those responses which were definitely initiated between 100 and $400 \mathrm{~ms}$ after stimulus presentation were included to the test data set comprising a total number of 587 responses.

As a major handicap of real data, the true response onset $t_{0}$ is unknown and, therefore, the onset error $\varepsilon=\hat{t}_{0}-t_{0}$ as used before cannot be determined. But performance of the methods can be assessed on a relative basis; to this, the onset error $\varepsilon=\hat{t}_{0}-t_{\text {ref }}$ was computed as the deviation between the onset estimates $\hat{t}_{0}$ provided by the tested methods and the

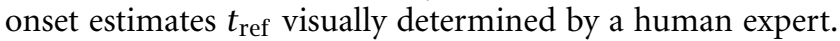
In order to maximize the percentage of correct detection of the methods, their decision thresholds were set individually by taking the smallest value $h$ for which the number of detected responses with alarm times $-50 \leq t_{\mathrm{a}}-t_{\text {ref }} \leq 200 \mathrm{~ms}$ was maximum. The resulting thresholds are summarized in Table 3.

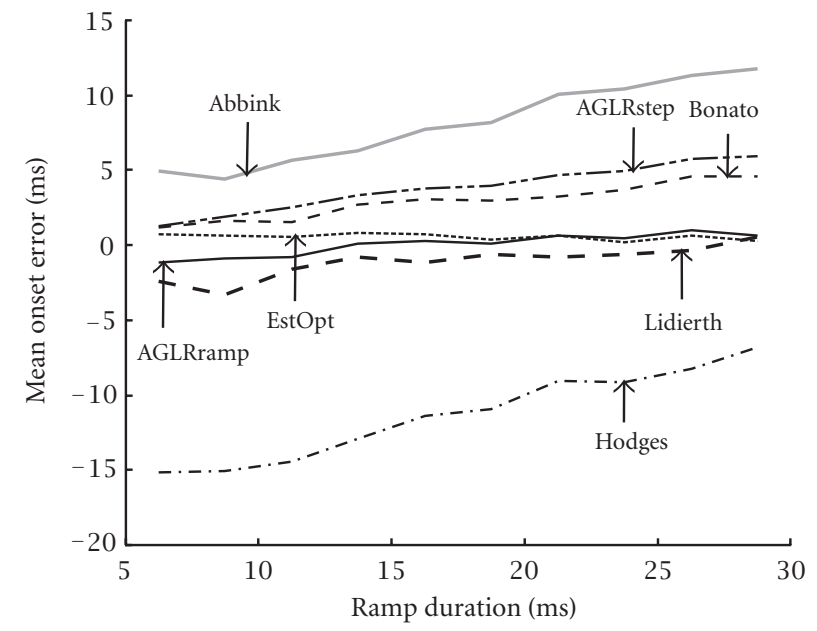

Figure 7: Dependence of mean onset error on ramp duration. Evaluation of Mixed Ramp Trials data set (SNR of $10 \mathrm{~dB}$, ramp duration 5-30 ms).

TABLE 3: Statistics for real SEMG data set.

\begin{tabular}{lcccr}
\hline Method & Threshold $h$ & $\begin{array}{c}\text { Detected } \\
\text { onsets }\end{array}$ & $\begin{array}{c}\text { Mean } \\
\text { error }\end{array}$ & $\begin{array}{r}\text { Error } \\
\text { STD }\end{array}$ \\
\hline AGLRramp & 200 & $100.0 \%$ & $0.4 \mathrm{~ms}$ & $3.6 \mathrm{~ms}$ \\
AGLRstep & 200 & $100.0 \%$ & $0.5 \mathrm{~ms}$ & $3.5 \mathrm{~ms}$ \\
Bonato & 20 & $99.8 \%$ & $-0.8 \mathrm{~ms}$ & $5.5 \mathrm{~ms}$ \\
Lidierth & 3 & $99.5 \%$ & $-2.3 \mathrm{~ms}$ & $6.9 \mathrm{~ms}$ \\
Abbink & 30 & $98.6 \%$ & $-0.6 \mathrm{~ms}$ & $9.8 \mathrm{~ms}$ \\
Hodges & 5 & $99.0 \%$ & $-7.5 \mathrm{~ms}$ & $9.3 \mathrm{~ms}$ \\
\hline
\end{tabular}

Figure 8 depicts two representative SEMG recordings. Onset estimates obtained with each method are indicated by markers. If the SEMG signal shows a large SNR and a steep change profile, all methods provide onset estimates close to the expert reference (see Figure 8a). If SNR decreases, onset estimates across methods will become more variable (see Figure $8 \mathrm{~b}$ ). The performance of the methods in real data onset detection is depicted by histograms of the onset error (see Figure 9a) showing the deviations of the computerized methods from the expert's reference and by the corresponding accuracy functions (see Figure 9b). Mean errors and standard deviations are summarized in Table 3. Consistently, the majority of SEMG recordings of this particular paradigm had large SNR and steep slopes like the sample recording in Figure 8a. This is reflected by the very narrow error histograms (see Figure 9a) and the rapidly increasing accuracy functions (see Figure 9b) indicating satisfactory performance for the majority of methods tested. Only the Hodges method provided onset estimates which were significantly smaller ( $7.5 \mathrm{~ms}$ on average) than the expert's decision, as indicated by the skewed error distribution (see Figure 9a). The ranking of methods is consistent with the ranking already obtained in the simulation study attesting highest performance to the GLR-based methods followed by the Bonato approach. Note 


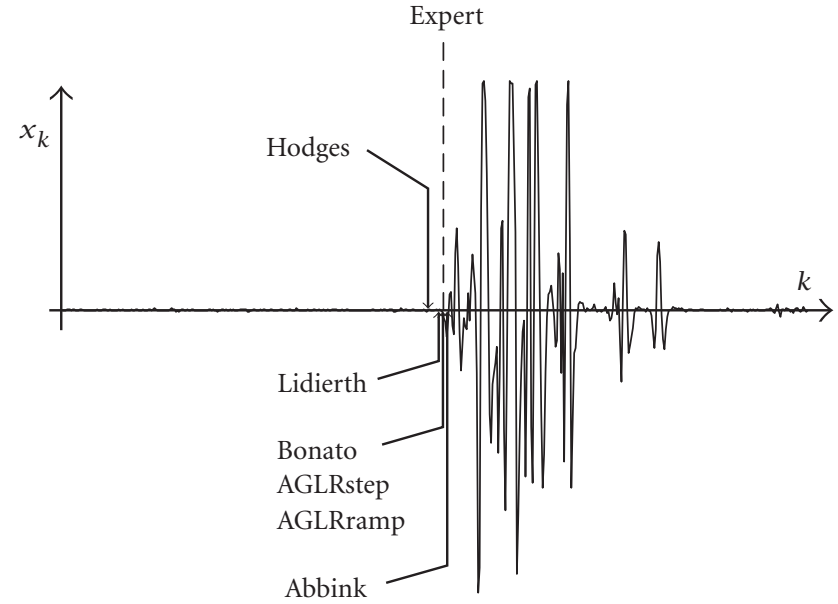

(a)

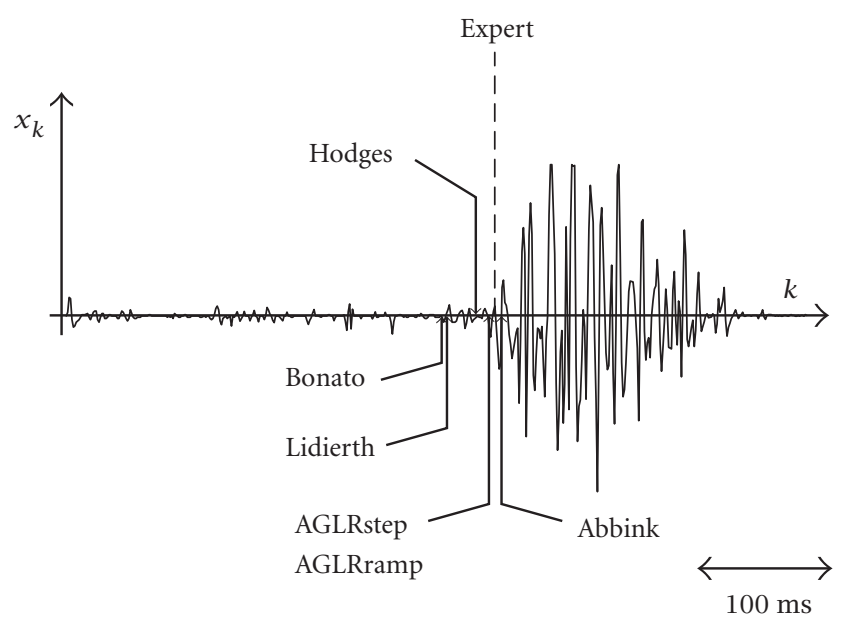

(b)

FIGURE 8: Raw SEMG signals obtained in a reaction time experiment: two sample traces (a) and (b) with different SNR. Onset estimates of each method are indicated by markers. Dotted lines indicate the expert's reference.

the small difference between the AGLRramp and AGLRstep algorithm indicating steep response profiles close to the steplike pattern of assumed by the AGLRstep method.

\section{DISCUSSION}

All methods perform well in detecting the onset time in good quality SEMG signals; thus, the difficulty to decide on the appropriate method appears with low SNR when SEMG signals are disturbed for some reason. In any case, the choice is also dependent on implementation issues like method complexity, real-time implementation capability, and the required CPU performance.

Simple threshold-based methods are very popular because of their intuitive and easy implementable structure. In

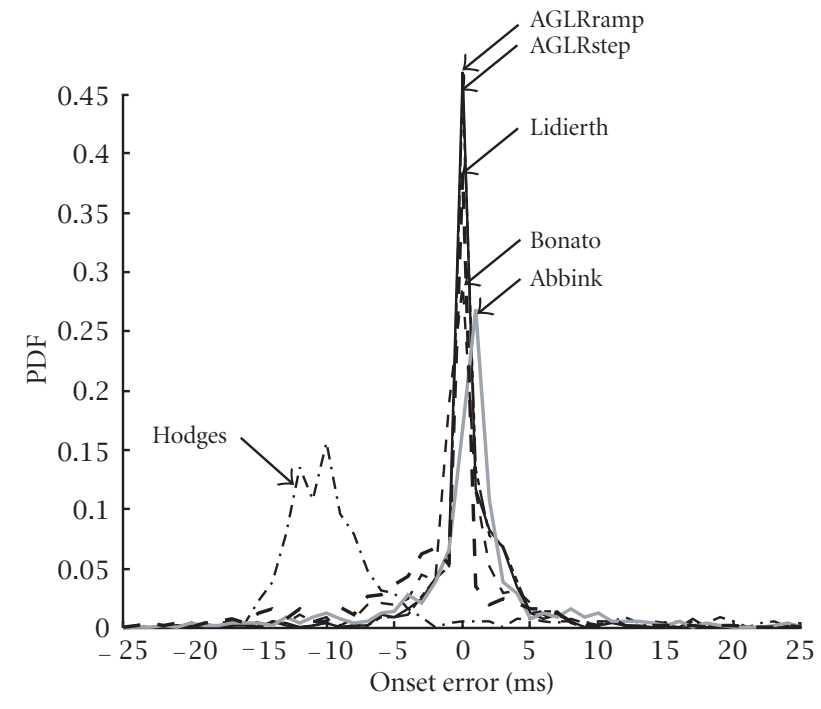

(a) Probability density functions of onset estimation errors.

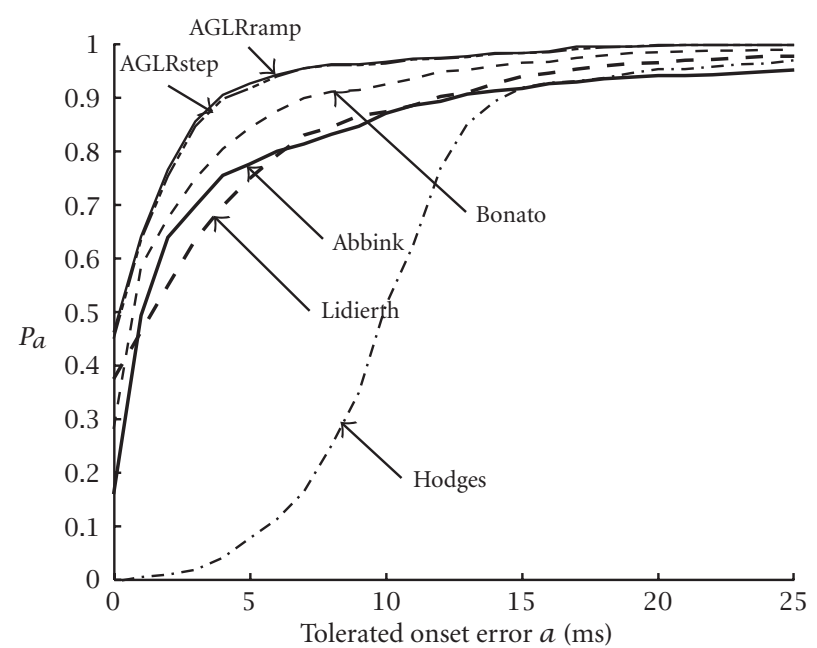

(b) Accuracy functions.

FIGURE 9: Analysis of deviations between estimated change times and expert's reference obtained on real SEMG signals with different computerized detectors.

most cases, SEMG signals have a good SNR, so these methods are well applicable with the main limitation that SNR should be larger than $10 \mathrm{~dB}$. But SEMG of small and deep muscles as well as SEMG recorded in patients with neuromuscular diseases may not fit into this SNR requirement, which calls for application of more sophisticated approaches.

Generally, purely threshold-based methods represent a tradeoff between detection precision and detection probability. A relatively low threshold level results in early onset detection but also causes more false alarms. High threshold levels, however, will usually lead to delayed or even missed onset detection. Although this delay can be partly compensated by subtracting an appropriate constant value from the 
alarm time, the large variability of resulting change time estimates and their systematic dependence on change dynamics confine the application of simple threshold-based methods if SNR is low and/or highly accurate change time estimation is required.

Analysis of simulated as well as real SEMG data showed that appropriate signal conditioning of the measured raw SEMG is a prerequisite for high detection performance. Lowpass filtering the (full-wave-rectified) SEMG before computing the test function substantially reduces the risk of false alarms. But it causes a very smooth incline of the pre-processed signal near the onset and thus will lead to increased variability of estimated change times. Simplethreshold detectors like Hodges are known for their results being very sensitive to the parameters of the lowpass filter used [13]. The Abbink approach suffers from this dilemma, too, despite of the more sophisticated change time estimation procedure.

Application of an adaptive pre-whitening filter proved to be superior to the less specific lowpass filter. Simulations with white Gaussian SEMG signals have shown that Bonato and Lidierth almost have the same onset error distribution, which is a consequence of their resembling detection strategies. Analysis of the more realistic colored SEMG data (see Figure 4) as well as analysis of real SEMG signals (see Figure 8) demonstrated the distinct performance gain of Bonato due to the use of an appropriate pre-whitening filter. Generally, the implicit highpass characteristic of the whitening filter preserves or even improves change dynamics as desired but accentuates the higher frequencies of possibly superimposed additive noise [22]. Therefore, we can expect good performance of the whitening filter provided that the noise is small compared to the variance profile $\sigma^{2}(k)$.

If higher robustness to changes in signal parameters and high detection precision is required, a pre-whitening filter together with a statistically optimized decision rule is the first choice. Consistently, the two model-based approaches AGLRstep and AGLRramp provided higher detection power and more accurate change time estimates than the unspecific detectors both for the simulated and real SEMG signals. Particularly, these methods were most robust with respect to variations in signal properties such as SNR and change dynamics. However, model-based approaches are usually associated with higher computational efforts, particularly, when the dynamic of the change is not known and has to be estimated from the data. Results show only minor loss of performance for the computationally more efficient AGLRstep algorithm compared to the more specific but more complex AGLRramp algorithm. In order to reduce the computational effort of such more complex parametric detectors, a step-like stopping rule according to (31) may be combined with the ML change time estimator as shown in (37). This allows for an efficient implementation of the detection procedure, since the time-consuming estimation of the onset shape profile is only initiated once a change has been indicated.

An important aspect of computerized onset detection is post-processing. Generally, combining an arbitrary stopping rule with a post-processor testing multiple alarm/change times for their plausibility can improve detection performance. Particularly, the detection threshold can be reduced since false alarms can be partly compensated by the postprocessor. The use of an adequate post-processor is particularly important when the SEMG signal contains multiple changes indicating different levels of muscle activation. In this case, the detection unit produces a sequence of change times indicating possible transitions between different levels of muscle activation. A post-processor then groups the segments according to their variance merging segments with similar variance together. Combinations of GLR-based decision rules and post-processor have been successfully applied for the detection of pre-motor silent periods in SEMG signals [22] and for the detection and classification of events in the uterine EMG [23]. But any of the post-processors presented is also dependent on signal conditioning and alarm time generation. This means that any impairment caused by signal conditioning will nevertheless affect detection.

\section{EPILOGUE: DESIGN, PRESENTATION, AND COMPARISON OF ALGORITHMS}

"An algorithm must be seen to be understood and the best way to learn how an algorithm works is to play with it." (Donald Knuth, in "The Art of Computer Programming," [24]).

Every algorithm has embedded parameters, and it works only properly if these parameters are appropriately chosen. "Good" parameters are often determined by trial and error using simulated data. Despite the importance of finding and choosing the right parameters, usually little emphasis is devoted to this step by papers about algorithms, presumably because there is no clear mathematical theory behind it (i.e., scientifically accepted).

The presentation of algorithms to the scientific community is usually done by writing about

(1) the practical and mathematical background,

(2) a useful class of models,

(3) a description of the algorithm(s),

(4) a theoretical analysis of the behavior in some limiting cases,

(5) a table of numbers showing the performance of the algorithm tested on simulated data, while keeping some parameters in the signal model and the algorithm fixed,

(6) as in (4), but also with information about competing algorithms,

(7) a plot of a signal together with the corresponding output of the algorithms in question.

What typically is missing is a tool

(a) to check and interactively change the signal and to find good parameters for the algorithms for other applications,

(b) to compare different algorithms with arbitrary combinations of parameters, 
(c) to check the behavior of the algorithm(s) with real data.

These aspects are even more valid in the case of on-line algorithms. In the statistical community, new ways to present results using the internet were emphasized [25], and several groups are currently working on software tools which allow a user with any standard browser to visualize and change signals and algorithms. To our experience, systematically but inductive trying of all the parameters in both the model and the algorithm yields quickly an idea about interesting neighborhoods in parameter space, quality, and stability of an algorithm. Software tools appropriate for this purpose should be easy to use and should not require a comprehensive installation procedure: neither many researchers nor referees do have the time to reprogram a new algorithm in their own favorite programming environment to evaluate new signal processing procedures. To illustrate this point, an onset detection program designed in MATLAB ${ }^{\circledR}$ will be made available to the readers by the authors, which allows to visualize the model described above, to change its parameters and to see the output of some selected algorithms. Using such tools simplifies to assess an algorithm or method, but requires to own a MATLAB ${ }^{\circledR}$ license up to now. But there are some developments to overcome this problem (e.g., compiler version) in near future, which will allow the software package to be made available through the internet.

\section{APPENDIX}

In this section, the implementation of the AGLRstep and AGLRramp decision rules for the present signal model are shortly described.

The structure of the tests depends upon the variance profiles before and after the response onset $t_{0}$ which, according to the process model, are

$$
\begin{aligned}
\sigma_{0}^{2}(k) & =\sigma_{\text {noise }}^{2} \\
\sigma_{1}^{2}\left(k, t_{0}\right) & =\sigma_{\text {noise }}^{2}+\sigma_{\text {signal }}^{2} u\left(k, t_{0}\right),
\end{aligned}
$$

respectively. The variance pattern $\sigma_{0}^{2}(k)$ before change (i.e., response onset) depends upon a single parameter

$$
\sigma_{0}^{2}\left(k, \theta_{0}\right)=\theta_{0}=\text { constant }
$$

which is equal to the unknown variance $\sigma_{\text {noise }}^{2}$ The $\mathrm{ML}$ estimate of $\theta_{0}$ can be obtained from the initial $M$ data points according to

$$
\hat{\theta}_{0}=\arg \sup _{\theta_{0}} \sum_{i=1}^{M}-\frac{1}{2}\left(\ln \theta_{0}+\frac{y_{i}^{2}}{\theta_{0}}\right) .
$$

Differentiation with respect to $\theta_{0}$ and solving the resulting likelihood equation

$$
\frac{1}{2}\left[\frac{1}{\theta_{0}^{2}} \sum_{i=1}^{M} y_{i}^{2}-\frac{M}{\theta_{0}}\right]=0
$$

leads to

$$
\sigma_{0}^{2}\left(k, \hat{\theta}_{0}\right)=\hat{\theta}_{0}=\frac{1}{M} \sum_{i=1}^{M} y_{i}^{2}
$$

Thus, the estimated variance profile before change is equal to the average signal energy within the reference window.

Determination of the variance pattern $\sigma_{1}^{2}(k, j)$ after change is more complicated since it depends upon the dynamic change profile $u(k, j)$ which, generally, is not known. This issue is addressed in the next paragraphs.

\section{AGLRstep detector}

The AGLRstep detector simply assumes an approximately constant variance profile of unknown magnitude $\theta_{1}$ after change, that is,

$$
\sigma_{1}^{2}\left(k, j, \theta_{1}\right)=\theta_{1}=\text { const. }
$$

In this case, the log-likelihood ratio in (14) can be written as

$$
\hat{S}_{j}^{k}=\sup _{\theta_{1}} \frac{1}{2} \sum_{i=j}^{k}\left[\left(\hat{\theta}_{0}^{-1}-\theta_{1}^{-1}\right) y_{i}^{2}+\ln \frac{\hat{\theta}_{0}}{\theta_{1}}\right],
$$

where $\hat{\theta}_{0}$ is the estimated variance before change according to (26). Maximization with respect to $\theta_{1}$ is explicitly possible by replacing $\theta_{1}$ by its ML estimate

$$
\hat{\theta}_{1}(j, k)=\frac{1}{k-j+1} \sum_{i=j}^{k} y_{i}^{2},
$$

which leads to

$$
\hat{S}_{j}^{k}=\frac{k-j+1}{2}\left(\frac{\hat{\theta}_{1}(j, k)}{\hat{\theta}_{0}}-\ln \frac{\hat{\theta}_{1}(j, k)}{\hat{\theta}_{0}}-1\right) .
$$

Thus, the AGLRstep decision rule can be summarized as

$$
\begin{gathered}
t_{\mathrm{a}}=\min \left\{k \geq W: g_{k} \geq h\right\}, \\
g_{k}=\hat{S}_{k-W+1}^{k}, \\
\hat{S}_{j}^{k}=\frac{k-j+1}{2}(\hat{\rho}(j, k)-\ln \hat{\rho}(j, k)-1), \\
\hat{t}_{0}=\arg \max \hat{S}_{j}^{t_{\mathrm{a}}+\Delta},
\end{gathered}
$$

where

$$
\hat{\rho}(j, k)=\frac{\hat{\theta}_{1}(j, k)}{\hat{\theta}_{0}}=\frac{(1 /(k-j+1)) \sum_{i=j}^{k} y_{i}^{2}}{(1 / M) \sum_{i=1}^{M} y_{i}^{2}}
$$

denotes the ratio between the estimated variances before (reference window) and after change (test window), respectively. This decision rule can be implemented in a completely recursive manner. The AGLRstep algorithm was implemented with parameters $W=25, h=10, \Delta=100$, and $M=200$. 


\section{AGLRramp detector}

The AGLRramp detector uses a more complex test function which also takes the dynamic change profile into account. It assumes that the shape $u(k, j)$ of the additive change in the variance profile is known but its exact magnitude $\sigma_{\text {signal }}^{2}$ is unknown, that is,

$$
\sigma_{1}^{2}\left(k, j, \theta_{1}\right)=\theta_{0}+\theta_{1} u(k, j) .
$$

Then, the log-likelihood ratio in (14) can be rewritten as

$$
\begin{array}{r}
\hat{S}_{j}^{k}=\sup _{\theta_{1}} \frac{1}{2} \sum_{i=j}^{k}\left[\left(\frac{1}{\hat{\theta}_{0}}-\frac{1}{\theta_{1} u(i, j)+\hat{\theta}_{0}}\right) y_{i}^{2}\right. \\
\left.+\ln \frac{\hat{\theta}_{0}}{\theta_{1} u(i, j)+\hat{\theta}_{0}}\right],
\end{array}
$$

where the unknown magnitude $\theta_{1}$ is the only parameter to be determined. Differentiating the log-likelihood ratio with respect to $\theta_{1}$ results in a likelihood equation which cannot be explicitly solved. Therefore, $\theta_{1}$ is estimated from the observed sequence according to

$$
\hat{\theta}_{1}(j, k)=\frac{\sum_{i=j}^{k}\left(y_{i}^{2}-\hat{\theta}_{0}\right)}{\sum_{i=j}^{k} u(i, j)}
$$

by exploiting the statistical independence of the data sequence. The resulting AGLR decision rule for the detection of an additive change with known dynamic profile but unknown magnitude is

$$
\begin{aligned}
t_{\mathrm{a}} & =\min \left\{k \geq W: g_{k} \geq h\right\}, \\
g_{k} & =\hat{S}_{k-W+1}^{k}, \\
\hat{S}_{j}^{k} & =\frac{1}{2} \sum_{i=j}^{k}\left[\left(s \frac{1}{\hat{\theta}_{0}}-\frac{1}{\hat{\theta}_{1} u(i, j)+\hat{\theta}_{0}}\right) y_{i}^{2}\right. \\
& \left.\quad+\ln \frac{\hat{\theta}_{0}}{\hat{\theta}_{1} u(i, j)+\hat{\theta}_{0}}\right], \\
\hat{\theta}_{1}(j, k) & =\frac{\sum_{i=j}^{k}\left(y_{i}^{2}-\hat{\theta}_{0}\right)}{\sum_{i=j}^{k} u(i, j)}, \\
\hat{t}_{0} & =\arg \max _{W \leq j \leq t_{\mathrm{a}}} \hat{S}_{j}^{t_{\mathrm{a}}+\Delta},
\end{aligned}
$$

where $\hat{\theta}_{1}(j, k)$ is individually determined for each combination $(j, k)$ according to $(35)$.

If the exact profile is also unknown, the test may include more maximizations which determine the ML estimates of the unknown parameters, too. Particularly, $u(k, j)$ may be replaced by a set of $N$ template profiles $u(k, j)^{(n)}$, $n=1,2, \ldots, N$, together with an additional maximization which selects the most likely template according to

$$
\begin{array}{r}
\hat{S}_{j}^{k}=\max _{1 \leq n \leq N} \frac{1}{2} \sum_{i=j}^{k}\left[\left(\frac{1}{\hat{\theta}_{0}}-\frac{1}{\hat{\theta}_{1}^{(n)} u(i, j)^{(n)}+\hat{\theta}_{0}}\right) y_{i}^{2}\right. \\
\left.+\ln \frac{\hat{\theta}_{0}}{\hat{\theta}_{1}^{(n)} u(i, j)^{(n)}+\hat{\theta}_{1}}\right] .
\end{array}
$$

The AGLRramp algorithm was implemented with parameters $W=25, h=10, \Delta=100, M=200$, and a set of $N=8$ shaping functions with ramp durations $\tau=5,10, \ldots, 40 \mathrm{~ms}$.

\section{REFERENCES}

[1] E. A. Clancy and N. Hogan, "Probability density of the surface electromyogram and its relation to amplitude detectors," IEEE Trans. Biomed. Eng., vol. 46, pp. 730-739, 1999.

[2] J. H. Abbink, A. van der Bilt, and H. W. van der Glas, "Detection of onset and termination of muscle activity in surface electromyograms," J. Oral Rhabil, vol. 25, pp. 365-369, 1998.

[3] S. V. Adamovich, M. F. Levin, and A. G. Feldman, "Merging different motor patterns: coordination between rhythmical and discrete single-joint movements," Exp. Brain Res., vol. 99, pp. 325-337, 1994.

[4] P. Brodin, T. S. Miles, and K. S. Turker, "Simple reactiontime responses to mechanical and electrical stimuli in human masseter muscle," Arch. Oral Biol., vol. 38, pp. 221-226, 1993.

[5] S. A. V. M. Haagh, W. A. C. Spijkers, B. Van den Boogaart, and A. Van Boxtel, "Fractioned reaction time as a function of response force," Acta. Psychol., vol. 66, pp. 21-35, 1987.

[6] J. K. Leader 3rd, J. R. Boston, and C. A. Moore, "A data dependent computer algorithm for the detection of muscle activity onset and offset from EMG recordings," Electroenceph. Clin. Neurophysiol., vol. 109, pp. 119-123, 1998.

[7] S. Micera, A. M. Sabatini, and P. Dario, "An algorithm for detecting the onset of muscle contraction by EMG signal processing," Med. Eng. Phys., vol. 20, pp. 211-215, 1998.

[8] J. Nilsson, M. Panizza, and P. Arieti, "Computer-aided determination of the silent period," J. Clin. Neurophysiol, vol. 14, pp. 136-143, 1997.

[9] R. D. Rafal, A. Winhoff, J. H. Friedman, and E. Bernstein, "Programming and execution of sequential movements in Parkinson's disease," J. Neurol. Neurosurg. Psychiatry, vol. 50, pp. 1267-1273, 1987.

[10] K. Takada and K. Yashiro, "Automatic measurement of on/off periods of EMG activity," Medinfo, vol. 8, pp. 751-754, 1995.

[11] G. J. M. van Boxtel, L. H. D. Geerats, M. M. C. Van den BergLenssen, and C. H. M. Brunia, "Detection of EMG onset in ERP research,” Psychophysiology, vol. 30, pp. 405-412, 1993.

[12] G. Staude and W. Wolf, "Objective motor response onset detection in surface myoelectric signals," Med. Eng. Phys., vol. 21, pp. 449-467, 1999.

[13] P. W. Hodges and B. H. Bui, "A comparison of computer-based methods for determination of onset of muscle contraction using electromyography," Electroenceph. Clin. Neurophysiol., vol. 101, pp. 511-519, 1996.

[14] P. Bonato, T. D’Alessio, and M. Knaflitz, "A statistical method for the measurement of muscle activation intervals from surface myoelectric signal during gait," IEEE Trans. Biomed. Eng., vol. 45, pp. 287-298, 1998.

[15] M. Lidierth, "A computer based method for automated measurement of the periods of muscular activity from an EMG and its application to locomotor EMGs," Electroenceph. Clin. Neurophysiol., vol. 64, pp. 378-380, 1986. 
[16] G. Staude, R. Dengler, and W. Wolf, “The discontinuous nature of motor execution part I: a model concept for single-muscle multiple-task coordination," Biol. Cybern., vol. 82, pp. 23-33, 2000.

[17] L. Ljung, System Identification: Theory for the User, PrenticeHall, New Jersey, 1987.

[18] G. Barrett, H. Shibasaki, and R. Neshige, "A computer-assisted method for averaging movement-related cortical potentials with respect to EMG onset," Electroenceph. Clin. Neurophysiol., vol. 60, pp. 276-281, 1985.

[19] H. G. Choi, J. C. Principe, A. A. Hutchison, and J. A. Wozniak, "Multiresolution segmentation of respiratory electromyographic signals," IEEE Trans. Biomed. Eng., vol. 41, pp. 257-266, 1994.

[20] G. Staude, W. Wolf, and U. Appel, "Automatic event detection in surface EMG of rhythmically activated muscles," An Int. Conf. IEEE BME Soc., vol. 17, pp. 1351-1352, 1995.

[21] G. Staude and W. Wolf, "Voluntary motor reactions: does stimulus appearance prolong the actual tremor period?," J. Electromyogr. Kinesiol., vol. 9, pp. 277-281, 1999.

[22] G. Staude, V. Kafka, and W. Wolf, "Determination of premotor silent periods from surface myoelectric signals," Biomed. Tech., vol. 45, no. 2, pp. 228-232, 2000.

[23] M. Khalil and J. Duchêne, "Uterine EMG analysis: a dynamic approach for change detection and classification," IEEE Trans. Biomed. Eng., vol. 47, pp. 748-756, 2000.

[24] D. E. Knuth, The Art of Computer Programming, AddisonWesley, Massachusetts, 1998.

[25] W. West, T. Ogden, and A. Rossini, "Statistical tools on the WWW," The American Statistician, vol. 52, pp. 257-262, 1998.

Gerhard Staude received the M.S.E.E. in 1990 from the Technical University Munich, Germany, and the Ph.D. degree in electrical engineering in 1996 from the University of the Armed Forces at Munich, Germany. From 1996 to 1997 he was a member of the interdisciplinary graduate college "Sensory Interaction in Biological and Technical Systems" of the Deutsche Forschungsgemeinschaft. Currently, he is affiliated with the Department of

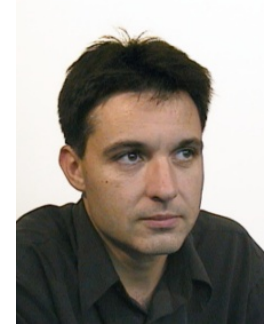
Electrical Engineering at the University of the Armed Forces, where he heads the Neuromuscular system research group at the Institute of Mathematics and Computer Science. He is author of more than 30 refereed journal papers in the fields of digital signal processing and analysis of the human motor system. His major research interests include parameter estimation and change detection in motorrelated biosignals, and basic research in the human motor system with special focus on biological oscillators (e.g., physiological and pathological tremors) and movement coordination.

Claus Flachenecker received his M.S.E.E in 1994. He continued as a Ph.D. student at BMW AG, Munich, in cooperation with the Technical University Munich, Lehrstuhl für Elektrische Meßtechnik. This work comprised intense cooperation with the European STAUMECS and German ASAM standardization groups, that define plug \& play integration standards for product testing environments. After receiving his Ph.D. degree

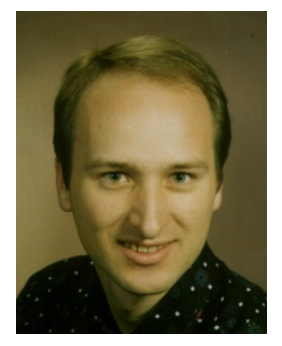
in 1998, he was affiliated with the University of the Armed Forces at Munich to research biosignal related topics, set-up a driving simulator environment with incorporated physiological driver monitoring and create intelligent force-feedback controllers to be used in forcefeedback pedals for automobiles. Since end of 2000, he is working for Carl Zeiss Ophthalmic, Inc. (California, USA), as a systems design engineer. His responsibilities now include the design and development of diagnostic medical devices for the eye-care industry.

Martin Daumer received the diploma in Physics in 1990 and the Ph.D. in mathematics both from the Ludwig-MaximiliansUniversity Munich, Germany in 1995. In 1987 he was a summer student at CERN, Geneva, and in 1992 he spent one year with a stipend of the DAAD at the Department for Mathematics at the Rutgers University, New Jersey. From 1993-1996 he was a member and later post-doc of the interdisciplinary

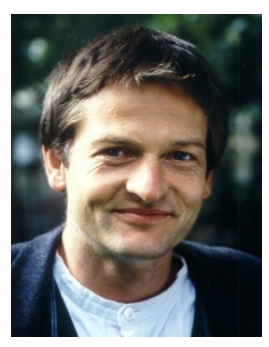
graduate college "Mathematics in its relationship with Physics" from the Deutsche Forschungsgemeinschaft DFG. Since 1996 he is heading the Research group "Online Monitoring in Medicine" in cooperation with the Sonderforschungsbereich 386 "Statistical Analysis of Discrete Structures" at the Institute for Medical Statistics and Epidemiology of the Technical University of Munich. He has published more than 40 articles about topics in quantum physics, scattering theory, probability theory, biosignal processing and holds patents for methods and devices for change-point detection and internetbased monitoring of biosignals. In 1999 he founded the IT-company Trium Analysis Online, which is playing a central role in various national (BMBF) and international research projects, for example, the Sylvia Lawry Centre for Multiple Sclerosis Research.

Werner Wolf received his M.S.E.E. in 1970 and his Ph.D. in 1978, both from the Technical University Munich, Germany. Now, he is a member of the Faculty of Electrical Engineering of the University of the Armed Forces at Munich. From 1970 to 1978, he was engaged in an interdisciplinary research project "Visual Perception" of the Institute of Communication Systems, Technical University Munich, with special focus on the func-

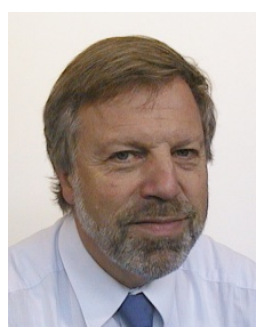
tional role of saccadic eye movements in vision. In 1978, he moved to the Institute of Mathematics and Computer Science now heading the Biosignal Processing Lab. His main interests are processing of biosignals (e.g., EP, EEG, EMG) and include basic research in sensorimotor systems (eye movements, hand and arm movements, etc.). Much of his research concerns pathophysiological aspects and is performed in cooperation with clinical departments. He is author of more than 100 scientific publications, and a member of the IEEE Society of Engineering in Medicine and Biology, the IEEE Society of Signal Processing, the American Society of Neuroscience, and several national engineering societies. 